\title{
On Bayesian Estimation of Loss and Risk Functions
}

\author{
Randhir Singh \\ Department of Statistics, Ewing Christian College, Prayagraj, India
}

Email address:

dr.singh.ecc@gmail.com

\section{To cite this article:}

Randhir Singh. On Bayesian Estimation of Loss and Risk Functions. Science Journal of Applied Mathematics and Statistics. Vol. 9, No. 3, 2021, pp. 73-77. doi: 10.11648/j.sjams.20210903.11

Received: June 7, 2021; Accepted: June 21, 2021; Published: June 26, 2021

\begin{abstract}
Loss functions and Risk functions play very important role in Bayesian estimation. This paper aims at the Bayesian estimation for the loss and risk functions of the unknown parameter of the H(r, theta), (theta being the unknown parameter) distribution The estimation has been performed under Rukhin's loss function. The importance of this distribution is that it contains some important distributions such as the Half Normal distribution, Rayleigh distribution and Maxwell's distribution as particular cases. The inverse Gamma distribution has assumed as the prior distribution for the unknown parameter theta. This prior distribution is a Natural Conjugate prior distribution for the unknown parameter because the posterior probability density function of the unknown parameter is also inverse gamma distribution The Rukhin's loss function involves another loss function denoted by w(theta, delta) he form of w(theta, delta) is important as it changes the estimate. In this paper, three forms of w(theta, delta) have been taken and corresponding estimates have been derived. The three, forms are, the Squared Error Loss Function (SELF) and two different forms of Weighted Squared Error Loss Function (WSELF) namely, the Minimum Expected Loss (MELO) Function and the Exponentially Weighted Minimum Expected Loss (EWMELO) Function have been considered. A criterion of performance of various form of w(theta, delta) has ben defined. It has been proved that among three forms of w(theta, delta), considered here, the form corresponding to EWMELO is most dominant.
\end{abstract}

Keywords: Bayes Estimator, Loss Function, Risk Function, H(r, theta) Distribution

\section{Introduction}

A continuous random variable $X$ is said to have $H(r, \theta)$ distribution, if its probability density function is given by,

$$
f(x, \theta)=\left\{\begin{array}{c}
\frac{2 \theta^{-\frac{r}{2}} x^{r-1} e^{-\frac{x^{2}}{\theta}}}{\Gamma\left(\frac{r}{2}\right)}, \text { if } x>0, \theta>0, r>0 \\
0, \text { Otherwise. }
\end{array}\right.
$$

This distribution covers some important distribution for various values of $r$. For example, $r=1,2$ and 3 respectively, the probability density function in (1) coincides with that of the probability density functions of Half Normal distribution, Rayleigh distribution and Maxwell's distribution respectively. This distribution used by Singh [1], the author for Bayesian estimation of Shannon's entropy and distribution function. It is to be noted that the random variable $\mathrm{Y}=\mathrm{X}^{2}$ follows gamma distribution with its probability density function is given by,

$$
g(y, \theta)=\left\{\begin{array}{c}
\frac{\theta^{-\frac{r}{2} x^{\frac{1}{2}-1} e^{-\frac{x}{\theta}}}}{\Gamma\left(\frac{r}{2}\right)}, \text { if } y>0, \theta>0, r>0 \\
0, \text { Otherwise. }
\end{array}\right.
$$

Rayleigh Distribution and Maxwell's distribution (which correspond to $\mathrm{r}=1$ and $\mathrm{r}=2$, respectively), were studied by many authors. Bhattacharya [2] introduced Bayesian approach in estimation of life testing using SELF. Tyagi and Bhattacharya [3-5] studied the classical and Bayesian estimation of Rayleigh and Maxwell's distribution. They also used only SELF for Bayesian estimation.. Chaturvedi and Rani [6] studied the reliability of the generalized Maxwell's distribution in the Bayesian framework. SELF and two forms of WSELF was used by Singh [7-8] in the study of reliability of a multicomponent system and Bayesian Estimation of the mean and distribution function of Maxwell's distribution under the assumption of conjugate prior distribution. Poddar and Roy [11] also studied Maxwell's distribution using modified linear exponential loss (MLINEX) function. Bekker 
and Roux [12] also considered some aspects of Maxwell's distribution in the light of Bayesian estimation. Day and Sudhanshu obtained Bayes estimators of parameters of Maxwell distribution bu using non-informative as well as conjugate prior distributions. They used quadratic loss function, SELF and MLINEX loss function. Krishna and Malik [14] have adopted progressive Type II censoring in order to estimate reliability of Maxwell's distribution. Xu et al. [15] considered the Bayesian inference for the loss and risk functions of parameter of Rayleigh distribution. Ajami and Jahanshahi [16] studied parameter estimation of Weighted Rayleigh Distribution in the classical as well as in Bayesian framework. Teena et al. [19, 20]. Have recently considered Bayesian estimation for Exponentiated Inverted Weibull distribution an Logarithmic Transformed Exponential distribution under different loss functions.

\section{Basic Concepts}

Guobing Fan [9] has derived the Bayes estimator of the loss and risk function of Maxwell's distribution using inverse Gamma distribution as the prior distribution for $\theta$ and squared error loss function under the criterion of loss function proposed by Rukhin [10], which is given by

$$
\mathrm{L}(\theta, \delta, \gamma)=\mathrm{w}(\theta, \delta) \gamma^{-\frac{1}{2}}+\gamma^{\frac{1}{2}}
$$

Where $\gamma$ is an estimator of the loss function $\mathrm{w}(\theta, \delta)$ which is non-negative.

$$
\frac{\partial \mathrm{L}(\theta, \delta, \gamma)}{\partial \gamma}=0 \text { gives } \gamma=\mathrm{w}(\theta, \delta)
$$

Since $\frac{\partial^{2} \mathrm{~L}(\theta, \delta, \gamma)}{\partial \gamma^{2}}>0$ at $\gamma=\mathrm{w}(\theta, \delta)$. So $\mathrm{L}(\theta, \delta, \gamma)$ is minimum in $\gamma$ when $\gamma=\mathrm{w}(\theta, \delta)$.

Let $\underline{X}=\left(X_{1}, X_{2}, \ldots X_{n}\right)$ be a random sample of size $n$, then the Bayes risk is

$$
\begin{gathered}
\mathrm{E}\{\mathrm{L}(\theta, \delta, \gamma) / \underline{\mathrm{X}}\}=\mathrm{E}\{\mathrm{w}(\theta, \delta) / \underline{\mathrm{X}}\} \gamma^{-\frac{1}{2}}+\gamma^{\frac{1}{2}} \\
\frac{\partial \mathrm{E}\{\mathrm{L}(\theta, \delta, \gamma) / \underline{\mathrm{X}}\}}{\partial \gamma}=0 \text { gives } \gamma=\mathrm{E}\{\mathrm{w}(\theta, \delta) / \underline{\mathrm{X}}\} .
\end{gathered}
$$

So, if $\delta_{\mathrm{B}}(\underline{\mathrm{X}})$ is the Bayes estimator of $\theta$ ) under the loss function $w(\theta, \delta) \gamma_{B}(X)$ be the Bayes estimator of $\theta$ corresponding to $\mathrm{L}(\theta, \delta, \gamma)$. Thus,

$$
\gamma_{\mathrm{B}}(\underline{\mathrm{X}})=\mathrm{E}\left\{\mathrm{w}\left(\theta, \delta_{\mathrm{B}}\right) / \underline{\mathrm{X}}\right\}
$$

Let $\underline{X}=\left(X_{1}, X_{2}, \ldots X_{n}\right)$ be a random sample of size $n$ from the distribution specified by the probability density function given by (1). Let $\mathrm{T}=\sum_{\mathrm{i}=1}^{\mathrm{n}} \mathrm{X}_{\mathrm{i}}^{2}$. Then $\mathrm{T}$ is sufficient statistic for the unknown parameter $\theta$ in (1). Since $X_{i}^{2}$ has gamma distribution having probability density function given by (2), by virtue of reproductive property of the gamma distribution, $\mathrm{T}=\sum_{\mathrm{i}=1}^{\mathrm{n}} \mathrm{X}_{\mathrm{i}}^{2}$ also follows gamma distribution and its probability density function is given by,

$$
g(t, \theta)=\left\{\begin{array}{c}
\frac{\theta^{-\frac{n r}{2} t^{\frac{n r}{2}}-1} e^{-\frac{t}{\theta}}}{\Gamma\left(\frac{n r}{2}\right)}, \text { if } t>0, \theta>0, r>0 \\
0, \text { Otherwise. }
\end{array}\right.
$$

Since the family of gamma distribution is complete, $\mathrm{T}$ is complete sufficient statistic for $\theta$. Since, $E(T)=\left(\frac{r n}{2}\right) \theta, \frac{2 T}{r n}$ is UMVUE for $\theta$. It is easy to verify that $\frac{2 \mathrm{~T}}{\mathrm{rn}}$ is also the Maximum Likelihood Estimator of $\theta$.

On the part of loss function, we have considered the Following:

(i) Squared Error Loss Function (SELF): In this case,

$$
\mathrm{w}(\theta, \delta)=(\theta-\delta)^{2}
$$

This loss function is symmetric but is unbounded.

(ii) Weighted Squared Error Loss Function (WSELF): In this case

$$
\mathrm{w}(\theta, \delta)=\theta^{-2}(\theta-\delta)^{2}
$$

This loss function is asymmetric but is bounded.

This form of loss function was used by Tummala and Sathe [17] and by Zellner [18]

(iii) Exponentially Weighted Squared Error Loss Function (EWSELF): In this case

$$
\mathrm{w}(\theta, \delta)=\theta^{-2} \mathrm{e}^{-\mathrm{a} \theta^{-1}}(\theta-\delta)^{2}, \mathrm{a}>0
$$

This form of loss function is also asymmetric and bounded. This form of loss function was used for the first time by Singh [1], the author in his work for D. Phil.

\section{Bayesian Estimation of Loss and Risk Function}

In this section, the estimation of the loss function has been performed for the probability density function specified by (1) under various forms of $w(\theta, \delta)$, given by (6), (7) and (8) respectively. Results are given in the following:

Theorem1. Let $\underline{X}=\left(X_{1}, X_{2}, \ldots X_{n}\right)$ be a random sample of size $n$ from the $H(r, \theta)$ distribution, specified by the probability density function given by (1). Under the assumption of inverse Gamma distribution as the prior distribution for $\theta$,Bayes estimators of $\theta$ corresponding to various loss functions given as above and Bayes estimators of $\mathrm{w}(\theta, \delta)$ based on Rukhin's loss function given by (3) are as follows:

(a) When $\mathrm{w}(\theta, \delta)=(\theta-\delta)^{2}$,

$$
\begin{gathered}
\hat{\theta}_{B}=\frac{\beta+T}{S+\alpha-1} \\
\gamma_{B}(\underline{X})=\frac{(\beta+T)^{2}}{(S+\alpha-2)(S+\alpha-1)^{2}} \\
E_{\theta}\left\{\gamma_{B}(\underline{X})\right\}=\frac{S \theta^{2}+(S \theta+\beta)^{2}}{(S+\alpha-2)(S+\alpha-1)^{2}}
\end{gathered}
$$

The risk function of $\hat{\theta}_{\mathrm{B}}$ is, 


$$
\mathrm{R}\left(\theta, \widehat{\theta}_{\mathrm{B}}\right)=\frac{\mathrm{S} \theta^{2}+\{(1-\alpha) \theta+\beta\}^{2}}{(\mathrm{~S}+\alpha-1)^{2}}
$$

(b) When $\mathrm{w}(\theta, \delta)=\theta^{-2}(\theta-\delta)^{2}$

$$
\begin{gathered}
\hat{\theta}_{M}=\frac{\beta+T}{S+\alpha+1} \\
\gamma_{M}(\underline{X})=\frac{1}{(S+\alpha+1)} \\
E_{\theta}\left\{\gamma_{M}(\underline{X})\right\}=\frac{1}{(S+\alpha+1)} \\
R\left(\theta, \hat{\theta}_{M}\right)=\frac{S+\left(\beta \theta^{-1}-\alpha-1\right)^{2}}{(S+\alpha+1)^{2}}
\end{gathered}
$$

(c) When $\mathrm{w}(\theta, \delta)=\theta^{-2} \mathrm{e}^{-\mathrm{a} \theta^{-1}}(\theta-\delta)^{2}, \mathrm{a}>0$

$$
\begin{gathered}
\hat{\theta}_{\mathrm{E}}=\frac{\beta+\mathrm{T}+\mathrm{a}}{\mathrm{S}+\alpha+1} \\
\gamma_{\mathrm{E}}(\underline{\mathrm{X}})=\frac{1}{(\mathrm{~S}+\alpha+1)}\left(\frac{\beta+\mathrm{T}}{\beta+\mathrm{T}+\mathrm{a}}\right)^{\mathrm{S}+\alpha} \\
\mathrm{E}_{\theta}\left\{\gamma_{\mathrm{E}}(\underline{\mathrm{X}})\right\}=\frac{1}{(\mathrm{~S}+\alpha+1)} \mathrm{E}\left\{\left(\frac{\beta+\mathrm{T}}{\beta+\mathrm{T}+\mathrm{a}}\right)^{\mathrm{S}+\alpha}\right\} \\
\mathrm{R}\left(\theta, \hat{\theta}_{\mathrm{E}}\right)=\frac{\mathrm{e}^{-\mathrm{a} \theta^{-1}}\left\{\mathrm{~S}+\left(\beta \theta^{-1}+\mathrm{a}^{-1}-\alpha-1\right)^{2}\right\}}{(\mathrm{S}+\alpha+1)^{2}}
\end{gathered}
$$

Where,

$$
\mathrm{S}=\frac{\mathrm{rn}}{2}
$$

Proof: Let the prior probability density function of $\theta$, which is inverse gamma distribution, be given by,

$$
\begin{aligned}
& \pi(\theta)=\left\{\begin{array}{c}
\frac{\beta^{\alpha} \theta^{-(\alpha+1)} \mathrm{e}^{-\frac{\beta}{\theta}}}{\Gamma(\alpha)}, \text { if } \theta>0, \alpha, \beta>0 \\
0, \text { Otherwise. }
\end{array}\right. \\
& \widehat{\theta}_{\mathrm{B}}=\mathrm{E}(\theta / \mathrm{T})=\frac{\beta+\mathrm{T}}{\mathrm{S}+\alpha-1} \\
& \gamma_{\mathrm{B}}(\underline{\mathrm{X}})=\mathrm{E}\left\{\mathrm{w}\left(\theta, \hat{\theta}_{\mathrm{B}}\right) / \underline{\mathrm{X}}\right\}=\mathrm{E}\left\{\left(\theta-\hat{\theta}_{\mathrm{B}}\right)^{2} / \mathrm{T}\right\} \\
& \operatorname{Var}(\theta / \mathrm{T})=\frac{(\beta+\mathrm{T})^{2}}{(\mathrm{~S}+\alpha-2)(\mathrm{S}+\alpha-1)^{2}} \\
& \mathrm{E}_{\theta}\left\{\gamma_{\mathrm{B}}(\underline{\mathrm{X}})\right\}=\frac{\mathrm{E}\left\{(\beta+\mathrm{T})^{2}\right.}{(\mathrm{S}+\alpha-2)(\mathrm{S}+\alpha-1)^{2}}=\frac{\mathrm{S} \theta^{2}+(\mathrm{S} \theta+\beta)^{2}}{(\mathrm{~S}+\alpha-2)(\mathrm{S}+\alpha-1)^{2}} \\
& \mathrm{R}\left(\theta, \hat{\theta}_{\mathrm{B}}\right)=\mathrm{E}_{\theta}\left\{\mathrm{w}\left(\theta, \hat{\theta}_{\mathrm{B}}\right)\right\}=\mathrm{E}_{\theta}\left\{\left(\theta-\frac{\beta+\mathrm{T}}{\mathrm{S}+\alpha-1}\right)^{2}\right\}=\frac{\theta^{2}\left\{\mathrm{~S}+(\alpha-1)^{2}\right\}+2 \beta(1-\alpha) \theta+\beta^{2}}{(\mathrm{~S}+\alpha-1)^{2}}=\frac{\mathrm{S} \theta^{2}+\{(1-\alpha) \theta+\beta\}^{2}}{(\mathrm{~S}+\alpha-1)^{2}}
\end{aligned}
$$

Remark 1. For $\mathrm{r}=3$, results as above, coincide with that given by Guobing Fan [9], for Maxwell distribution.

(b) When $\mathrm{w}(\theta, \delta)=\theta^{-2}(\theta-\delta)^{2}$, the Bayes estimator of $\theta$, denoted by $\hat{\theta}_{\mathrm{M}}$ is given by,

$$
\hat{\theta}_{M}=\frac{\int_{0}^{\infty} \theta^{-1} \pi(\theta / t) d \theta}{\int_{0}^{\infty} \theta^{-2} \pi(\theta) d \theta}
$$

After simplification, we get,

$$
\hat{\theta}_{M}=\frac{E\left(\theta^{-1} / T\right)}{E\left(\theta^{-2} / T\right)}=\frac{\beta+T}{S+\alpha+1}
$$




$$
\gamma_{M}(\underline{X})=E_{\theta}\left\{w\left(\theta, \hat{\theta}_{M}\right) / \underline{X}\right\}=E\left\{\theta^{-2}\left(\theta-\hat{\theta}_{M}\right)^{2} / T\right\}=\frac{1}{S+\alpha+1}
$$

Since, $\frac{1}{S+\alpha+1}$ is a constant, $\mathrm{E}\left\{\gamma_{\mathrm{M}}(\underline{\mathrm{X}})\right\}=\frac{1}{(\mathrm{~S}+\alpha+1)}$

$$
\mathrm{R}\left(\theta, \hat{\theta}_{\mathrm{M}}\right)=\mathrm{E}_{\theta}\left\{\mathrm{w}\left(\theta, \hat{\theta}_{\mathrm{M}}\right)\right\}=\mathrm{E}_{\theta}\left\{\theta^{-2}\left(\theta-\frac{\beta+\mathrm{T}}{\mathrm{S}+\alpha+1}\right)^{2}\right\}=\frac{\mathrm{S}+\left(\beta \theta^{-1}-\alpha-1\right)^{2}}{(\mathrm{~S}+\alpha+1)^{2}}
$$

(c)When $\mathrm{w}(\theta, \delta)=\theta^{-2} \mathrm{e}^{-\mathrm{a} \theta^{-1}}(\theta-\delta)^{2}$, a $>0$, the Bayes estimator of $\theta$, denoted by $\hat{\theta}_{\mathrm{E}}$ is given by,

$$
\hat{\theta}_{M}=\frac{\int_{0}^{\infty} \theta^{-1} \mathrm{e}^{-\mathrm{a} \theta^{-1}} \pi(\theta / \mathrm{t}) \mathrm{d} \theta}{\int_{0}^{\infty} \theta^{-2} \mathrm{e}^{-\mathrm{a} \theta^{-1}} \pi(\theta) \mathrm{d} \theta}
$$

After simplification, we get,

$$
\begin{gathered}
\hat{\theta}_{\mathrm{E}}=\frac{\mathrm{E}\left(\theta^{-1} \mathrm{e}^{\left.-\mathrm{a} \theta^{-1} / \mathrm{T}\right)}\right.}{\mathrm{E}\left(\theta^{-2} \mathrm{e}^{\left.-\mathrm{a} \theta^{-1} / \mathrm{T}\right)}==\frac{\beta+\mathrm{T}+\mathrm{a}}{\mathrm{S}+\alpha+1}\right.} \\
\gamma_{\mathrm{E}}(\underline{X})=\mathrm{E}\left\{\mathrm{w}\left(\theta, \hat{\theta}_{\mathrm{E}}\right) / \underline{X}\right\}=\mathrm{E}\left\{\theta^{-2} \mathrm{e}^{-\mathrm{a} \theta^{-1}}\left(\theta-\hat{\theta}_{\mathrm{E}}\right)^{2} / \mathrm{T}\right\}=\frac{1}{\mathrm{~S}+\alpha+1}\left(\frac{\beta+\mathrm{T}}{\beta+\mathrm{T}+\mathrm{a}}\right)^{S+\alpha}
\end{gathered}
$$

So, $E_{\theta}\left\{\gamma_{E}(\underline{X})\right\}=\frac{1}{(S+\alpha+1)} E_{\theta}\left\{\left(\frac{\beta+T}{\beta+T+a}\right)^{S+\alpha}\right\}$

Since, $0<\left(\frac{\beta+T}{\beta+T+a}\right)^{S+\alpha}<1,0<E_{\theta}\left\{\left(\frac{\beta+T}{\beta+T+a}\right)^{S+\alpha}\right\}<1$. Therefore,

$$
\begin{gathered}
\mathrm{E}_{\theta}\left\{\gamma_{\mathrm{E}}(\underline{\mathrm{X}})\right\}<\frac{1}{(\mathrm{~S}+\alpha+1)}=\mathrm{E}_{\theta}\left\{\gamma_{\mathrm{M}}(\underline{\mathrm{X}})\right\} \\
\mathrm{R}\left(\theta, \hat{\theta}_{\mathrm{E}}\right)=\mathrm{E}_{\theta}\left\{\mathrm{w}\left(\theta, \hat{\theta}_{\mathrm{E}}\right)\right\}=\mathrm{E}_{\theta}\left\{\theta^{-2} \mathrm{e}^{-\mathrm{a} \theta^{-1}}\left(\theta-\frac{\beta+\mathrm{T}+\mathrm{a}}{\mathrm{S}+\alpha+1}\right)^{2}\right\}=\frac{\mathrm{e}^{-\mathrm{a} \theta^{-1}}\left\{\mathrm{~S}+\left(\beta \theta^{-1}+\mathrm{a}^{-1}-\alpha-1\right)^{2}\right\}}{(\mathrm{S}+\alpha+1)^{2}}
\end{gathered}
$$

Definition: Let $\gamma_{1}(\underline{\mathrm{X}})$ and $\gamma_{2}(\underline{\mathrm{X}})$ be the two estimators based on Rukhin's loss function corresponding to two different forms of $\mathrm{w}(\theta, \delta) \cdot \gamma_{1}(\underline{\mathrm{X}})$ is said to dominate $\gamma_{2}(\underline{\mathrm{X}})$ if $\mathrm{E}_{\theta}\left\{\gamma_{1}(\underline{\mathrm{X}})\right\} \leq \mathrm{E}_{\theta}\left\{\gamma_{2}(\underline{\mathrm{X}})\right\}$

Since, $\quad \mathrm{E}_{\theta}\left\{\gamma_{\mathrm{E}}(\underline{\mathrm{X}})\right\}<\mathrm{E}_{\theta}\left\{\gamma_{\mathrm{M}}(\underline{\mathrm{X}})\right\}, \quad \gamma_{\mathrm{E}}(\underline{\mathrm{X}}) \quad$ dominates $\gamma_{\mathrm{M}}(\underline{\mathrm{X}})$.

Since, $\mathrm{E}_{\theta}\left\{\gamma_{\mathrm{B}}(\underline{\mathrm{X}})\right\}=\frac{\mathrm{S}^{2}+(\mathrm{S} \theta+\beta)^{2}}{(\mathrm{~S}+\alpha-2)(\mathrm{S}+\alpha-1)^{2}}>\frac{1}{(\mathrm{~S}+\alpha+1)}$, provided $\theta \geq 1$

Thus, $\mathrm{E}_{\theta}\left\{\gamma_{\mathrm{E}}(\underline{\mathrm{X}})\right\}<\mathrm{E}_{\theta}\left\{\gamma_{\mathrm{M}}(\underline{\mathrm{X}})\right\}$, for all $\alpha, \beta$, and $\theta$ and $\mathrm{E}_{\theta}\left\{\gamma_{\mathrm{M}}(\underline{\mathrm{X}})\right\}<\mathrm{E}_{\theta}\left\{\gamma_{\mathrm{B}}(\underline{\mathrm{X}})\right.$ for all $\beta$ and $\theta \geq 1$. Hence, $\gamma_{\mathrm{E}}(\underline{\mathrm{X}})$ is most dominant among $\gamma_{\mathrm{E}}(\underline{\mathrm{X}}), \gamma_{\mathrm{M}}(\underline{\mathrm{X}})$ and $\gamma_{\mathrm{B}}(\underline{\mathrm{X}})$

Remark 2. For $r=1,2$, and 3 respectively, we get results for the Half Normal distribution, Rayleigh distribution and Maxwell distribution respectively.

\section{Conclusion}

In this paper Bayesian estimation of loss and risk functions for the unknown parameter $\theta$ of $\mathrm{H}(\mathrm{r}, \theta)$ distribution has been considered under Rukhin's loss function for three different forms of $\mathrm{w}(\theta, \delta)$. This distribution contains Half Normal distribution, Rayleigh distribution and Maxwell distribution as special case. The superiority of estimates has also been studied and it has been proved that when $\mathrm{w}(\theta, \delta)=\theta^{-2} \mathrm{e}^{-\mathrm{a} \theta^{-1}}(\theta-\delta)^{2}, \mathrm{a}>0$, the corresponding estimate is most dominant.

\section{Acknowledgements}

The author gratefully acknowledges the helpful comments and suggestions of the reviewers which led to improvement of this work.

\section{Conflict of Interest}

There exists no conflict of interest.

\section{References}

[1] Singh, Randhir, 1997. D. Phil Thesis (Unpublished), Department of Mathematics and Statistics, University of Allahabad, Allahabad. (INDIA).

[2] Bhattacharya, S. K.., 1967. Bayesian Approach to Life Testing and Reliability Estimation, JASA 62, pp. 48-62.

[3] Tyagi, R. K.\& Bhattacharya, S. K., 1989. Bayes Esimation of Maxwell's Velocity Distribution Function, Statistica, anno XLIX, n. 4, pp. 563-567.

[4] Tyagi, R. K. \& Bhattacharya, S. K., 1989. A Note On the MVU Estimation of Reliability for the Maxwell's Failure Distribution, ESTADISTICA, 41, 137, pp. 73-79.

[5] Tyagi, R. K. \& Bhattacharya, S. K., 1990. Bayesian Survival Analysis Based on the Rayleigh Model, TRABAJOS DE ESTADISTICA, Vol. 5 Num. 1, pp. 81-92. 
[6] Chaturvedi, A. and Rani U., 1998. Classical and Bayesian reliability estimation of the generalized Maxwell failure distribution. Journal of Statistical Research, 32: 113-120.

[7] Singh, Randhir, 1999. Bayesian Analysis of a Multicomponent System, Proceedings of NSBA-TA, 16-18 Jan. 1999, pp. 252-261, Editor Dr. Rajesh Singh. The conference was organised by the Department of Statistics, Amrawati University, Amravati-444602, Maharashtra, India.

[8] Singh, Randhir, 2010. Simulation Aided Bayesian Estimation fo Maxwell's Distribution, Proceedings of National Seminar on Impact of Physics on Biological Sciences (August, 26, 2010), held by the Department of Physics, Ewing Christian College, Prayagraj, India, pp. 203-210; ISBN No.: 978-81-905712-9-6.

[9] Guobing Fan, 2016. Estimation of the Loss anf Risk Functions of Parameter of Maxwell Distribution. Science Journal of Applied Mathematics and Statistics Vol. 4 No. 4, 2016, pp 129-133. doi: 10.11648/j.sjams.20160404.12.

[10] Rukhin A. L. 1988. Estimating the loss of estimators of binomial parameter. Biometrica, 75 (1): 153-155.

[11] Poddar C. K. and Roy M. K. 2003. Bayesian estimation of the parameter of Maxwell distribution under MLINEX loss function, Journal of Statistical Studies, 23: 11-16.

[12] Bekker, A. and Roux, J. J., 2005. Reliability characteristics of the Maxwell distribution: a Bayes estimation study, Comm. Stat. Theory \& Meth., 34 (11): 2169-2178.

[13] Day S. and Sudhanshu, S. M., 2010. Bayesian estimation of the parameter of Maxwell distribution under different loss functions. Journal of Statistical Theory \& Practice, 4 (2): 279-287.
[14] Krishna H. and Malik M., 2011 Reliability estimation in Maxwell distribution with progressively Type-II censored data. Journal of Statistical Computation and Simulation, 82 (4): 1-19.

[15] Xu M. P., Ding X. Y. and Yu J., 2013 Bayes inference for the loss and risk functions of Rayleigh distribution parameter estimator. Mathematics in Practice \& Theory, 43 (21): $151-156$.

[16] Ajami, Masoud and Jahanshahi, Seyed Mahdi Amir, 2017Journal of modern applied statistical methods: JMASM November 2017. DOI: 10.22237/jmasm/1509495240.

[17] Tummala, V. M. and Sathe, P. T., 1978. Minimum Expected Loss Estimators of Reliability and Parameters of Certain Life Time Distributions. IEEE Transactions on Reliability, Vol. R-27, No. 4, pp. 283-285.

[18] Zellner, A. and Park, S. B. 1979. Minimum Expected Loss Estimators (MELO) of Functions of Parameters and Structural Coefficients of Econometric Models. JASA 74, pp. 185-193.

[19] Teena Goyal, Piyush Kant Rai and Sandeep K. Maurya. 2019. Bayesian Estimation for Exponentiated Weibull distribution under different Loss Functions. International Journal of Pure and Applied Researches. http://ijopar.com2019 Vol. 2 (1): pp. 01-13ISSN: 2455-474X

[20] Teena Goyal, Piyush Kant Rai and Sandeep K. Maurya. 2020. Bayesian Estimation for Logarithmic Transformed Exponential 1 distribution under different Loss Functions. Journal of Statistics Applications \& Probability. Vol 9 No. 1, pp. 139-148. http://dx.doi.org/10.18576/jsap/090113. 